\title{
Promoting adherence through a directly administered antiretroviral therapy strategy in Mombasa, Kenya
}

Avina Sarna

Population Council

Stanley Luchters

Scott Geibel

Population Council

Matthew F. Chersich

Paul Munyao

See next page for additional authors

Follow this and additional works at: https://knowledgecommons.popcouncil.org/departments_sbsr-hiv

Part of the Demography, Population, and Ecology Commons, Family, Life Course, and Society

Commons, International Public Health Commons, and the Medicine and Health Commons

How does access to this work benefit you? Let us know!

\section{Recommended Citation}

Sarna, Avina, Stanley Luchters, Scott Geibel, Matthew F. Chersich, Paul Munyao, Rick Homan, Susan Kaai, Kishorchandra N. Mandaliya, Marleen Temmerman, and Naomi Rutenberg. 2007. "Promoting adherence through a directly administered antiretroviral therapy strategy in Mombasa, Kenya," Horizons Research Summary. Nairobi: Population Council. 


\section{Authors}

Avina Sarna, Stanley Luchters, Scott Geibel, Matthew F. Chersich, Paul Munyao, Rick Homan, Susan Kaai, Kishorchandra N. Mandaliya, Marleen Temmerman, and Naomi Rutenberg 


\section{Promoting Adherence through a Directly AdMINISTEREd ANTIRETROVIRAL Therapy Strategy in Mombasa, Kenya}

A directly-administered antiretroviral therapy strategy (DAART) was more effective in promoting adherence during the 24-week intervention period than standard of care, but the effect was not sustained postintervention. DAART patients had greater improvements in body mass index and depression scores than non-DAART patients. Results suggest that $D A A R T$ is effective for improving adherence in this setting, but the optimal length of the intervention for sustaining adherence and its transition to standard care may warrant further research.

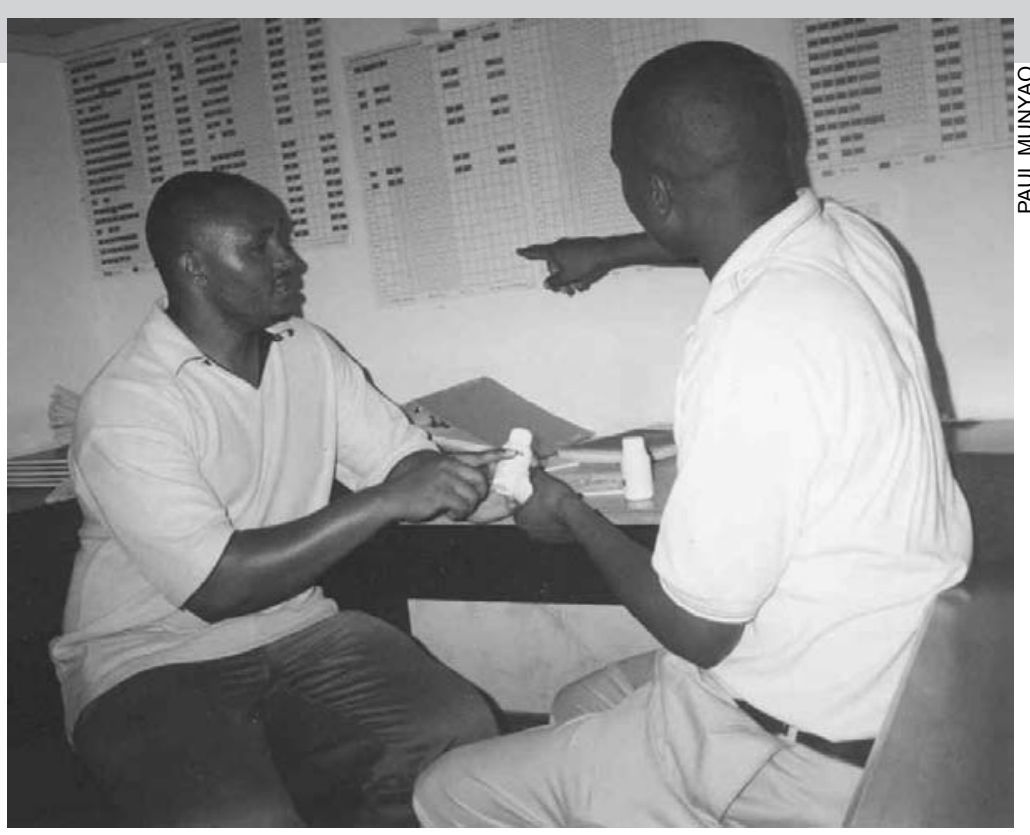

Jerry Okal (left), a key research assistant, with a community health worker who counsels ART patients on adherence.
$\Lambda$ principal concern of antiretroviral therapy (ART) programs is the ability of clients to maintain a high level of adherence to medication. Based on formative research conducted with HIV-infected clients and health workers in Mombasa, Kenya, and lessons learned from directly observed therapy (DOT) strategies to encourage adherence to treatment for tuberculosis, a DAART strategy to promote adherence to ART was developed. This study examined whether the DAART intervention was more effective in fostering adherence to ART than standard follow up among people living with HIV in Mombasa.

\section{Methodology}

Researchers from the Horizons Program and the International Centre for Reproductive Health, in collaboration with Coast Province General Hospital, Mkomani Bomu Clinic, and Port Reitz District Hospital, conducted a randomized, controlled, two-arm study to determine the short-term and longer-term effects of DAART compared to standard of care. Ethical approval for the study was obtained from the Ethical Review Board at Kenyatta National Hospital and the Institutional Review Board of the Population Council.

To read more about this study, go to www.popcouncil.org/horizons/projects/Kenya_HAARTMombasa.htm

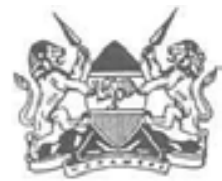


A total of $234 \mathrm{HIV}$-infected, treatment naïve patients who initiated highly active antiretroviral therapy (HAART) were enrolled in the study between September 2003 and November 2004 and were randomized to either the DAART arm or the standard of care arm. All patients received a first line non-nucleoside reverse transcriptase inhibitor (NNRTI) containing treatment regimen (stavudine, lamivudine and efavirenz or nevirapine) and were followed for 72 weeks. All patients received standard adherence counseling consisting of 3 one-on-one preparatory sessions prior to initiating HAART as well as ongoing needs-based counseling support at routine monthly clinic visits.

The DAART intervention lasted for a period of 24 weeks. During this time, participants in the DAART arm visited a health center twice a week where they met with DAART observers (nurses) who observed the ingestion of one dose of antiretroviral medications, performed pill-counts, collected used medication bottles, enquired about difficulties encountered, and provided individualized adherence support. At these visits medications were dispensed for the following three or four days, until the next visit. To enhance convenience, participants could select one of six health centers for their DAART visits. During the DAART intervention, community health workers (CHWs) traced participants who missed visits and carried medications home for those who, for reasons of ill-health, were unable to visit the center. After the first 24 weeks, DAART patients were followed by routine monthly visits for a further 48 weeks.

Non-DAART patients received standard monthly follow up for the entire 72 weeks. This involved adherence counselling and routine monthly follow-up clinic visits. CHWs traced DAART and non-DAART clients who did not keep the routine monthly follow-up clinic appointments.

Adherence was assessed using clinic-based pill counts that measured adherence over a four-week period. Pill counts were undertaken every four weeks for 48 weeks of follow up and again at 72 weeks. Adherence was calculated by dividing the number of pills actually taken by the number of pills the client was required to take over the reporting period multiplied by 100 . Adherence was dichotomized to $\geq 95$ percent and $<95$ percent. The primary indicator for adherence in this study was the proportion of patients able to achieve $\geq 95$ percent adherence over $0-24,25-48$ and at 72 weeks. A p value of $<0.05$ was considered significant. Self-reported adherence based on a 4-day recall of missed doses was also used to assess adherence.

Patients who did not complete at least 30 days in the study were excluded from the analysis. Participants known not to have collected medication from the pharmacy, or who had dropped out of the study were awarded $<95$ percent adherence for that reporting period and thereafter treated as missing data. Those who attended clinic visits but failed to bring back bottles were considered missing pill counts for that reporting period. Some came back with fewer pills than expected, possibly due to misplaced pills, repeated ingestion if pills had been vomited, or pill dumping. Fewer pills than expected produce values of adherence greater than 100 percent; in these instances patients were given an adherence value equal to 100 minus the excess percent adherence (e.g., 102 percent adherence was given 98 percent). Patients who died were considered to have $<95$ percent adherence for the reporting period immediately following their death and thereafter treated as missing.

A secondary outcome indicator for the study was the proportion of patients with undetectable virus $(<400$ copies $/ \mathrm{ml}$ ) at 48 and 72 weeks. High levels of adherence allow patients to achieve maximum treatment effectiveness and undetectable virus in the blood. Thus viral load is a proxy marker for adherence. Viral load testing was conducted at 48 weeks and 72 weeks.

Other health outcomes assessed in the study included CD 4 cell counts, body weight, and depression. CD4 counts, a measure of immunological function, and body weight are expected to rise with ART. Body mass index (weight in $\mathrm{kg} /$ height in $\mathrm{m}^{2}$ ) is a reliable indicator for body fat and nutritional status. To assess depression among study participants, the research- 
ers used the Beck Depression Inventory $\mathrm{I}^{\oplus}$, a 21 -item validated tool. The tool was culturally adapted to the Kenyan context before application. Changes in CD4 cell counts, body mass index (BMI), and depression scores were calculated for each patient with baseline and secondary measurements and the median change for each indicator was calculated for the group.

This summary presents the results from 72 weeks of follow up. The findings focus on adherence during the DAART intervention period (0-24 weeks) and on sustained adherence in the early post-intervention period (25-48 weeks) and on longer-term adherence after the intervention (72 weeks).

\section{Recruitment and Retention}

A total of 234 patients were recruited; 116 patients were randomized to the DAART arm and 118 to the non-DAART arm. Seven patients did not receive the allocated intervention due to death prior to starting ART $(\mathrm{n}=3)$, withdrawal $(\mathrm{n}=3)$, and severe illness $(\mathrm{n}=1)$. Thus 227 patients were eligible for adherence monitoring (DAART: 111; non-DAART: 116). Eighty-nine patients in the DAART arm and 94 in

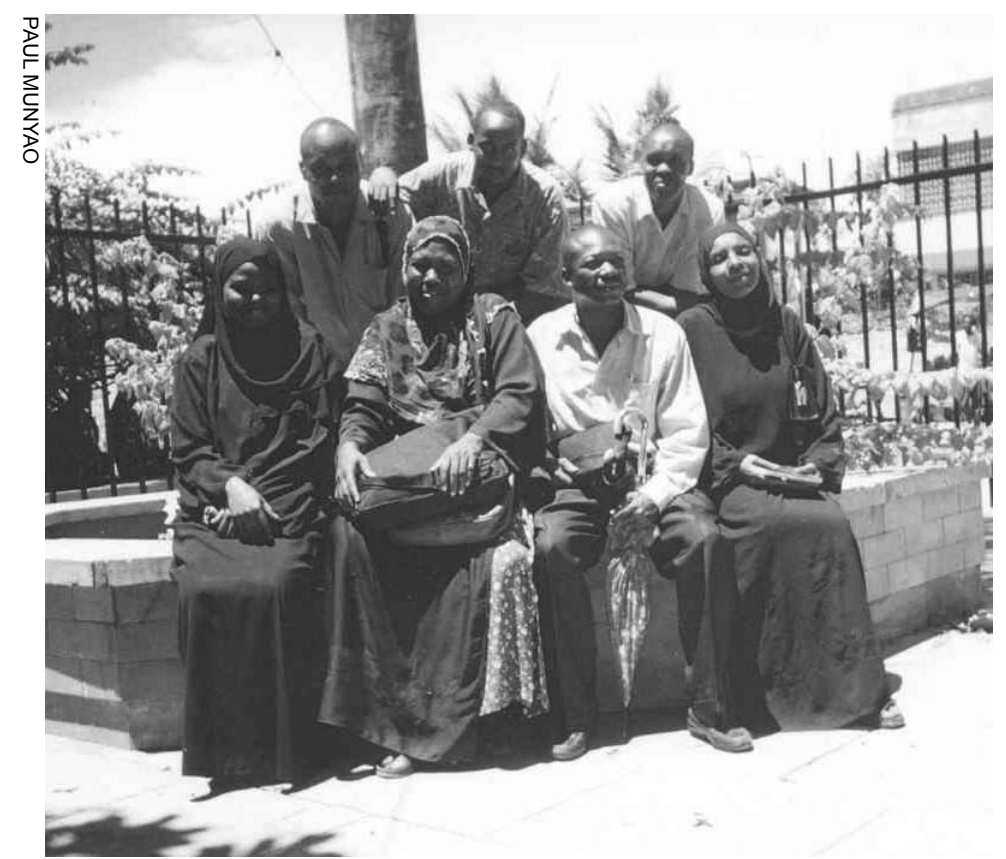

A team of community health workers who trace clients who do not appear for observed treatment or to pick up medication. the non-DAART arm completed 72 weeks of follow up. Twenty-five patients died before completing 72 weeks of follow up (DAART: 14; non-DAART: 10); the majority of these died in the first 24 weeks of follow up (67 percent). Ten patients discontinued the intervention: six patients transferred to other hospitals (DAART: 2; non-DAART: 4) and four non-DAART patients stopped treatment. Ten patients were lost to follow up, their whereabouts unknown (DAART: 6; non-DAART: 4).

Sociodemographic characteristics were similar across the two groups (Table 1). Almost two- thirds (64 percent) of patients were female, half of them had received primary education, and half were currently married. The median age was 36 years (IQR: 31-43). The median CD 4 cell counts were 99 cells $/ \mathrm{mm}^{3}$ (IQR 49-145) at baseline; nearly a quarter of the patients had CD4 cell counts less than 50 cells $/ \mathrm{mm}^{3}$ at the beginning of the study.

There were no significant differences with regard to age, sex, and CD4 counts between patients lost to follow up and patients completing the study. During the study one patient changed to a protease inhibitorbased treatment regimen (AZT, DDI, Lopinavir/ Ritonavir).

\section{Key Findings}

\section{The majority of patients made 90 percent or more of expected DAART visits.}

The DAART intervention required patients to make a total of 48 visits over 24 weeks of follow up to DAART observation sites to pick up their medication and be observed taking a dose. Arrangements were made to provide medications to patients who wanted to travel outside of Mombasa.

Service data from DAART sites show that 59 percent of DAART patients who completed 24 weeks of follow up $(n=94)$ attended 90 percent or more of DAART visits, 16 percent attended between 80 to 89 percent of required visits, and 20 percent attended between 50 to 79 percent of visits. Only 4 percent attended less than half of required visits. 


\section{The DAART group had higher adherence during the intervention period however, the effect was not sustained post-intervention.}

Data from pill counts show that a significantly higher proportion of patients in the DAART group were able to achieve adherence $\geq 95$ percent during the intervention period ( $0-24$ weeks; $\mathrm{p}=0.012)$ compared to patients in the control group (Figure 1). However the effect was not sustained and the difference between groups was not statistically significant both in the early post intervention period (25-48 weeks) and in the late post-intervention period (72 weeks). Adherence declined sharply in the DAART group in the early post intervention period when DAART supervision was withdrawn.

A similar trend was obtained with self reported adherence using a 4-day recall. A significantly smaller proportion of DAART patients reported missed doses during 0-24 weeks compared to the non-DAART patients ( 9 percent vs. 19 percent; $\mathrm{p}=0.04$ ). There were no significant differences in the post intervention period (25-48 wks: 13 percent vs. 13 percent; $\mathrm{p}=0.92$; 72 wks: 3 percent vs. 5 percent; $\mathrm{p}=0.49$ )

\section{Viral load suppression was not significantly greater among $D A A R T$ patients.}

More patients in the DAART arm achieved viral loads $<400$ copies $/ \mathrm{ml}$ at 48 weeks compared to those in the control group, but the difference was not statistically significant (88 percent vs. 78 percent). At 72 weeks, the figures for the two groups were similar (74 percent vs. 77 percent), but this was due to a decline in the number of patients in the DAART group who achieved undetectable viral loads (88 percent to 74 percent).

\section{Both groups demonstrated immunological improvement.}

At baseline, there were no significant differences in median CD 4 counts between groups. Patients in both groups with baseline and secondary measurements demonstrated improvements in median CD4 counts at 24,48 , and 72 weeks. But there was no significant difference in the median change in CD4 counts between the two groups over 0-24 weeks, 0-48 weeks, and $0-72$ weeks (Figure 2).

Figure 1 Proportion of patients with adherence $\geq 95$ percent in DAART and non-DAART groups

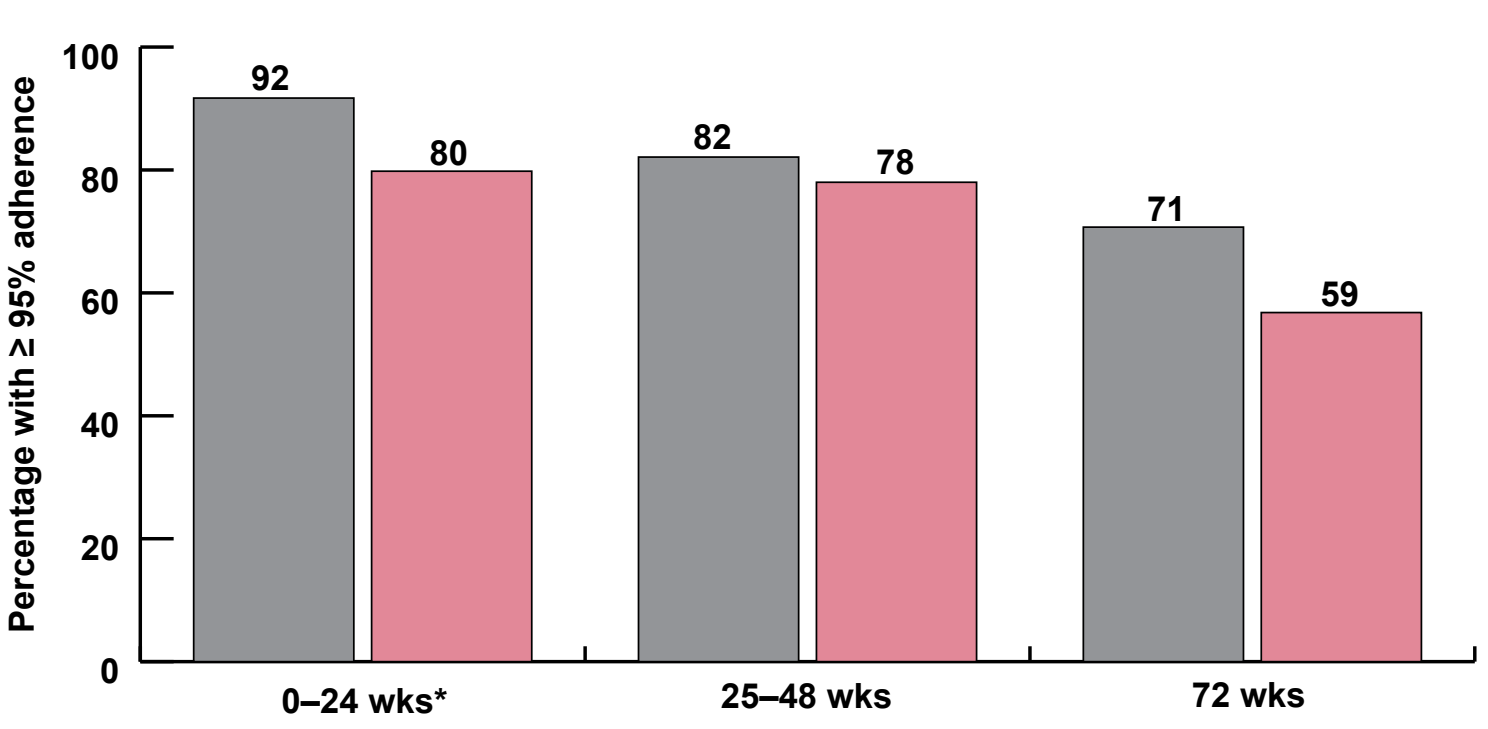

DAART

Non-DAART 
Figure 2 Baseline CD4 counts and median increase in CD4 counts in the study groups over 24, 48 and 72 weeks of follow up

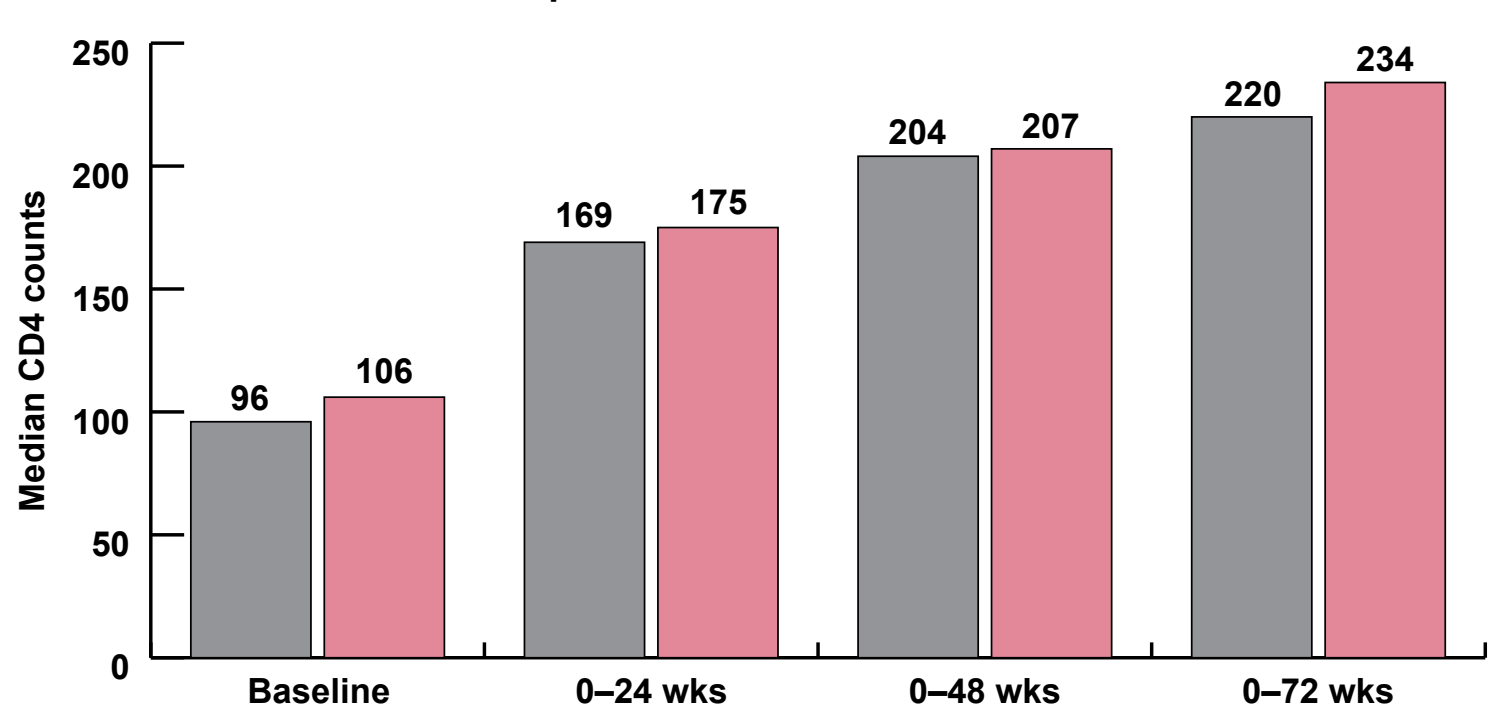

DAART

Non-DAART

DAART patients had greater improvements in body mass index.

There was no difference in BMI scores between the two groups at baseline (DAART: $20.6 \mathrm{~kg} / \mathrm{m}^{2}$ vs. nonDAART: $20.8 \mathrm{~kg} / \mathrm{m}^{2}$ ). Significant improvements in BMI were observed in both groups. Patients in the DAART group had a significantly greater mean increase in BMI over $0-24(\mathrm{p}=0.014)$ and $25-48(\mathrm{p}=$ 0.047) weeks compared to those in the control group. Although patients in the DAART group had a higher mean increase in BMI at 0-72 weeks compared to those in the control group, the difference was not statistically significant (Figure 3).

\section{Patients in the DAART group had greater improvement in depression scores}

At the beginning of the study, nearly a third of the patients in both groups reported moderate to severe depression (31.7 percent). There were no differences in depression scores between groups at baseline (DAART: 15.6 vs. non-DAART: $14.5 ; \mathrm{p}=0.23$ ).

Figure 3 Mean change in BMI in the study groups over 24, 48 and 72 weeks of follow up

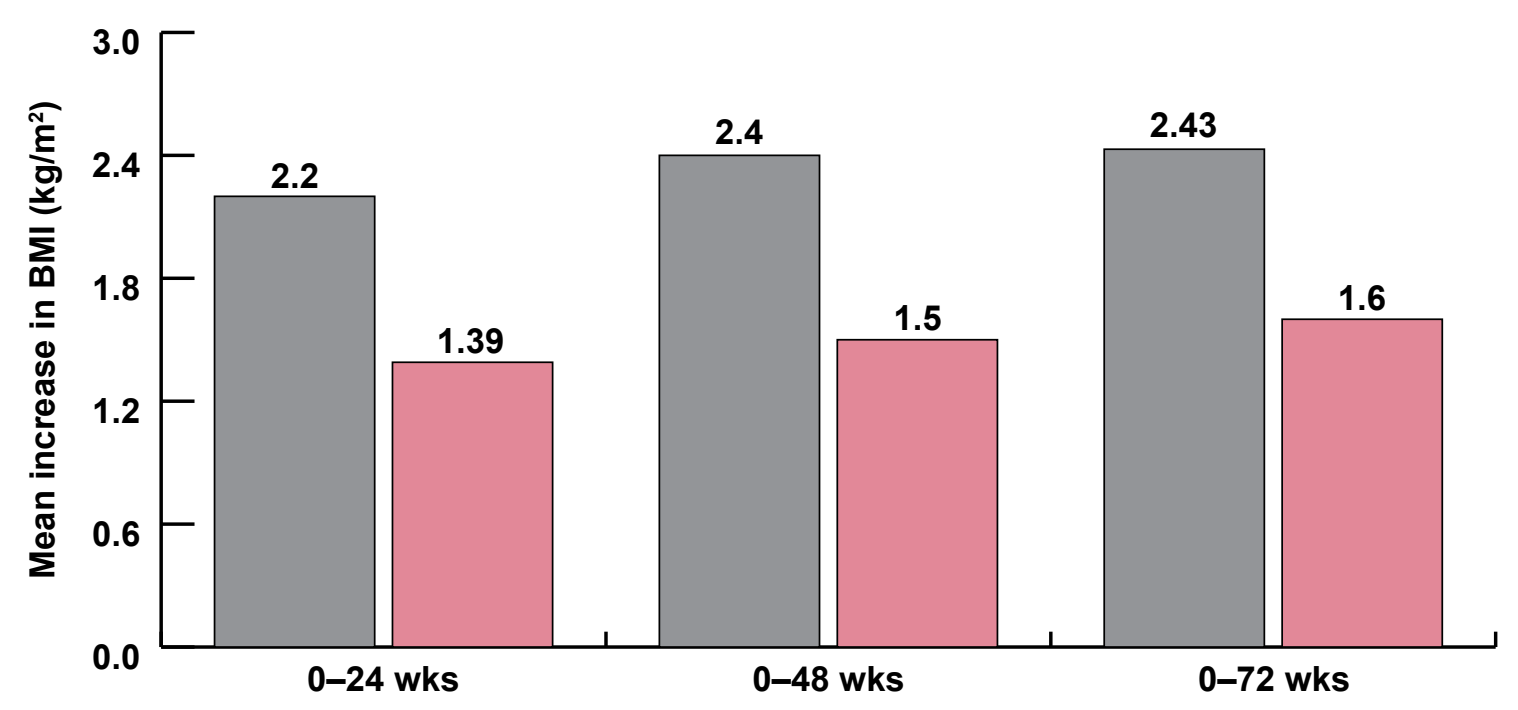

DAART

Non-DAART 
Figure 4 Median decrease in depresssion scores in the study groups over 24, 48 and 72 weeks of follow up

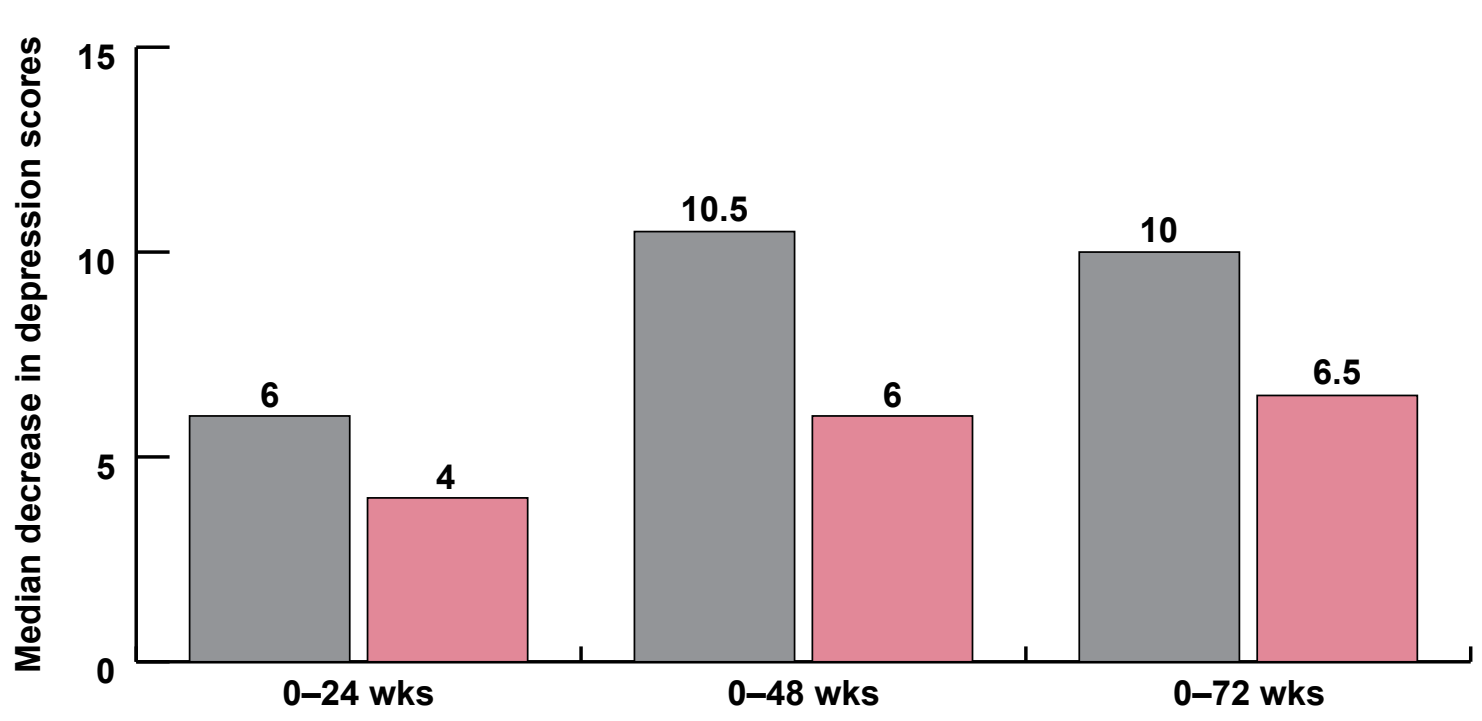

Both groups demonstrated significant reductions in depression scores over 72 weeks on treatment. The DAART group had a significantly larger median decrease in depression scores at $0-48$ weeks $(\mathrm{p}=0.04)$ and $0-72(\mathrm{p}=0.03)$ weeks, but not at $0-24$ weeks (Figure 4).

\section{DAART was particularly effective in promoting adherence among depressed patients.}

During the intervention phase (0-24 weeks), patients with moderate to severe depression in the DAART arm were seven times as likely to achieve $\geq 95$ percent adherence than comparably depressed patients in the control arm (OR = 7.0; 95\% CI: 2.7-18.0; $\mathrm{p}<0.001)$ [Data not shown]. But after week 24, there was no longer any difference in adherence levels among moderately to severely depressed patients by study arm.

\section{Conclusions}

High levels of adherence to HAART were observed for all patients during the first 24 weeks. DAART was more effective in promoting adherence during the intervention period ( $0-24$ weeks); a higher proportion of patients in the DAART group achieved $\geq 95$ percent adherence during the intervention period com- pared to those in the non-DAART group. DAART patients also had greater increases in BMI during the intervention period and from 0 to 48 weeks, and larger declines in depression scores at $0-48$ weeks and 0-72 weeks.

Study findings suggest that DAART may be particularly useful among patients with moderate to severe depression, which is common among HIV-infected persons (Ciesla and Roberts 2001). Depression has previously been shown to be an independent predictor of poor adherence (Paterson et al. 2000; Gordillo et al. 1999). Further, both non-adherence and depressive symptoms are associated with higher mortality among patients receiving ART (Lima et al. 2007). Though this finding needs to be confirmed, it suggests that DAART may be particularly useful for this high-risk subgroup.

However, the effects of the intervention on adherence were not sustained in the early and late post-intervention periods. Moreover, adherence declined over time in both groups, which is of concern to ART programs. The decline was sharper in the DAART group in the period following the cessation of the intervention, possibly due to the sudden withdrawal of adherence support. A gradual stepping down to standard care may have prevented this decline and needs to be considered by all programs using a modified DOT approach to promote adherence. 


\section{Estimated Incremental Annual Cost Per Patient of a Directly Administered Antiretroviral Therapy Program}

The incremental annual cost of a DAART program was determined by the value of the additional resources required to support the program. In the Mombasa program the additional resources required include: staff time at the health facility to observe the clients, staff time of the CHWs who served as trackers, and additional transportation and communication expenses for the trackers. The volume of additional resources required was estimated as part of a larger costing study of the ART program at Coast Province General Hospital (CPGH) conducted in 2004-05, which measured expenses from the previous year (2003-04).

Additional staff time at the health facility was estimated through a patient flow analysis of the comprehensive care center at CPGH. Contacts with health facility staff by DAART clients were compared to non-DAART clients to estimate the additional staff time required to support the DAART program. This additional staff time was then converted to a cost estimate based upon standard Ministry of Health payment rates for the different job categories at $\mathrm{CPGH}$.

Program records of monthly payments made to the CHWs to serve as trackers (1,000 Ksh per tracker per month) as well as the additional transportation and communication payments to trackers were used to estimate the incremental cost of the DAART program associated with the use of trackers.

Combining the value of the additional staff time at the health facility with the payments made to the trackers yielded the incremental cost of the DAART program. All costs were then inflated to an annual period and divided by the number of clients served in the project to yield the estimated annual cost per client for the DAART program.

\section{Results}

For the DAART program in Mombasa, Kenya, the annual incremental cost per client was 2,164 KSh per client ( USD31). The value of the additional health facility staff time accounted for 80 percent of the total cost while the payments to the $\mathrm{CHWs}$ for their tracking activities accounted for the remaining 20 percent (USD6.20). In terms of additional expenditures, only the payments to the CHWs for their efforts and their transportation and communication expenses represented additional monetary costs to the program.

A larger proportion of patients in the DAART group compared to the control group achieved undetectable viral loads at 48 weeks, although the difference between groups was not statistically significant. Higher adherence during the intervention period as a result of DAART may have had a beneficial effect extending beyond the intervention period. The larger decline over time in the proportion of patients achieving viral load suppression in the DAART group, compared to the control group, may have occurred as a result of lower adherence in the wake of the cessation of the DAART intervention.
It is of note that a quarter of the patients in both groups had detectable virus at 72 weeks indicating treatment failure, despite the fact that all patients were ART naïve and received first line NNRTI treatment regimens. This is of concern given that recent research suggests that patients receiving NNRTI treatment regimens may be able to achieve undetectable viral loads even with adherence levels lower than $\geq 95$ percent (Maggiolo et al. 2005; Weiser et al. 2004).

In conclusion, the DAART intervention was successful in promoting adherence during the intervention 
period but not after the intervention ended. Overall results suggest that DAART is effective within certain parameters, but the optimum duration of the intervention is unclear and ways to sustain the effects of the intervention warrant more research. $\mathbb{R}$

December 2007

\section{References}

Ciesla, J. A. and J. E. Roberts. 2001. "Meta-analysis of the relationship between HIV infection and risk for depressive disorders," American Journal of Psychiatry 158: 725-730.

Gordillo, V. et al. 1999. "Sociodemographic and psychological variables influencing adherence to antiretroviral therapy," AIDS 13: 1763-1769.

Lima, V. D. et al. 2007. "The effect of adherence on the association between depressive symptoms and mortality among HIV-infected individuals first initiating HAART," AIDS 21: 1175-1183.

Maggiolo, F. et al. 2005. "Similar adherence rates favor different virologic outcomes for patients treated with non-nucleoside analogues and protease inhibitors," Clinical Infectious Diseases 40: 158-63.

Paterson, D. L. et al. 2000. "Adherence to protease inhibitor therapy and outcomes in patients with HIV infection," Annals of Internal Medicine 133(1): 21-30.

Weiser, S. D. et al. 2004. "Higher rates of viral suppression with non-nucleoside reverse transcriptase inhibitors compared to single protease inhibitors are not explained by better adherence," HIV Clinical Trials 5(5): 278-87.
Investigators for this study were Stanley Luchters of the International Centre for Reproductive Health, Avina Sarna and Susan Kaai of Horizons/Population Council. Intervention and study partners include Horizons, ICRH, Coast Province General Hospital, Mkomani Bomu Clinic, Port Reitz District Hospital, FHI/IMPACT, MSH/RPM Plus and Kenya Ministry of Health.

For more information on this study please contact Avina Sarna (asarna@popcouncil.org) or Stanley Luchters (Stanley.Luchters@icrh.org).

Suggested citation: Sarna, Avina, Stanley Luchters, Scott Geibel, Matthew Chersich, Paul Munyao, Rick Homan, Susan Kaai, Kishor Mandaliya, Marleen Temmerman, and Naomi Rutenberg. 2007. "Promoting adherence through a directly administered antiretroviral therapy strategy in Mombasa, Kenya," Horizons Research Summary. Nairobi: Population Council.

\section{Hqrizons} Population Council/Horizons Communications Unit 4301 Connecticut Avenue, NW Suite 280 Washington, DC 20008
This research summary is made possible by the generous support of the American people through the United States Agency for International Development (USAID) and the President's Emergency Plan for AIDS Relief under the terms of HRN-A-00-97-00012-00. The contents are the responsibility of the Horizons Program and do not necessarily reflect the views of USAID or the United States Government. 